\title{
Subclinical acute kidney injury (AKI) due to iodine-based contrast media
}

\author{
Claudio Ronco • Fulvio Stacul • Peter A. McCullough
}

Received: 2 April 2012 /Revised: 31 May 2012 / Accepted: 8 June 2012 / Published online: 16 August 2012

(C) European Society of Radiology 2012

\begin{abstract}
Contrast-induced acute kidney injury (CI-AKI), previously known as contrast-induced nephropathy (CIN), is a syndrome in which an acute renal dysfunction is diagnosed after the intravascular injection of contrast media. AKI implies an injury or damage but not necessarily a reduction in overall renal filtration function. The renal damage becomes evident only when more than $50 \%$ of the renal mass is compromised. This typically occurs when AKI is diagnosed using creatinine as a marker; in fact, creatinine is a surrogate of glomerular filtration and it does not describe the whole spectrum of kidney function. Recent AKI classifications include even slight changes in serum creatinine (as low as $0.3 \mathrm{mg} / \mathrm{dl}$ ), which are associated with worse outcomes. An early diagnosis of AKI using novel biomarkers
\end{abstract}

\author{
C. Ronco \\ Department of Nephrology, International Renal Research Institute \\ (IRRIV), San Bortolo Hospital, \\ Vicenza, Italy \\ F. Stacul \\ S.C. Radiologia Ospedale Maggiore, Azienda Ospedaliero- \\ Universitaria Ospedali Riuniti, \\ Trieste, Italy \\ P. A. McCullough \\ St. John Providence Health System, \\ Warren, MI, USA \\ P. A. McCullough \\ St. John Hospital and Medical Center, \\ Detroit, MI, USA \\ P. A. McCullough \\ Providence Hospital and Medical Centers, \\ Southfield and Novi, MI, USA \\ F. Stacul $(\square)$ \\ S.C. Radiologia Ospedale Maggiore, \\ Piazza Ospitale 1, \\ 34125 Trieste, Italy \\ e-mail: fulvio.stacul@aots.sanita.fvg.it
}

has now become possible. These new biomarkers provide additional value, not only because they facilitate earlier diagnosis but also because they can diagnose AKI even in the absence of a change in subsequent filtration function. Thus, in this situation, these new criteria can reveal subclinical AKI. A new domain of AKI diagnosis could then include functional and structural criteria as indicated by laboratory testing.

Key Points

- There is continuing concern about renal damage caused by radiological contrast agents

- Acute kidney injury may be associated with minor changes in serum creatinine

- AKI implies damage but not necessarily a reduction in overall renal filtration function.

- Novel biomarkers facilitate earlier diagnosis, even if subsequent filtration function is unaltered.

- AKI diagnosis could include functional and structural criteria as indicated by laboratory testing

Keywords Contrast medium-induced nephropathy · Iodinebased contrast media $\cdot$ Acute kidney injury $\cdot$ Renal failure . Definitions

Contrast-induced acute kidney injury (CI-AKI), previously known as contrast-induced nephropathy (CIN), represents an important problem for radiologists. CI-AKI occurs in up to $15 \%$ of the general population receiving intravascular iodine-based contrast agents [1]. Intra-arterial administration in those with chronic kidney disease (CKD) with additional risk factors including diabetes, heart failure, anaemia, hyperglycemia, and haemodynamic instability heighten the risk to $\sim 50 \%$ with a probability of requiring renal replacement therapy during hospitalisation or rehospitalisation of $\sim 10 \%$ [2-4]. Although the incidence may be low in the overall population undergoing imaging diagnostic and therapeutic procedures, the absolute number of events may 
represent a significant burden, given the overall number of examinations performed in clinical routine worldwide. Furthermore, the risk with intravenous may be similar to intraarterial injections given a greater load of contrast agent balanced by more admixing with the blood pool and a greater water distribution in the body before being delivered to the kidneys by the renal arteries [5]. The epidemiology of this clinical entity has been reported in several papers exploring various populations with or without pre-existing risk factors for renal dysfunction. A significant variability in its incidence and severity has been described in different studied populations. This could be for various reasons including different baseline risk conditions, additional risk factors, different techniques utilised and different contrast media. However, one of the main sources of heterogeneity in population-based reports is the criteria utilised to define $\mathrm{AKI}$ and CIN.

$\mathrm{CI}-\mathrm{AKI}$ has been defined as an adverse event occurring in patients receiving contrast media for imaging procedures. It is characterised by a rapid deterioration of kidney function documented by a rise in serum creatinine ( $\mathrm{SCr}$ ) or cystatin $\mathrm{C}$, which may be transient or evolve into more severe and chronic kidney dysfunction. In general, the causal mechanism has been identified as transient vasoconstriction and ischaemia in a predisposed kidney with superimposed direct cellular toxicity to renal tubular cells [6]. Today, a new understanding of the AKI syndrome may require a revisitation of the CI-AKI or CIN definition, utilising harmonised, modern criteria to define kidney injury and dysfunction. The scope of this commentary is to drive the attention of the radiological community on the recent advances in the comprehension of the AKI syndrome and its new criteria for definition and staging.

AKI is a complex, time-variable syndrome with a multifactorial pathophysiology whose epidemiology and outcomes are variable in relation to the criteria utilised for detection and determination of severity. The concept of AKI has been evolving over recent years. Both its diagnosis and management have become multidisciplinary with major involvement of critical care medicine, cardiology, hepatology, radiology and other disciplines. With this evolution, the term ARF used for many years in clinical practice has been replaced with the term AKI. The new term implies a potentially reversible injury or damage to the kidney occurring in a timeframe of hours or days. This is mainly characterised by an abrupt decrease of glomerular filtration with consequent clinical derangements. Thus, while the term "injury" does not necessarily imply dysfunction, the diagnosis of AKI is still made on a change in $\mathrm{SCr}$ or a drop in urine output, as manifestations of an acute decline in glomerular filtration rate (GFR). Few studies have reported the results from biopsies carried out during AKI [7], demonstrating a variable involvement of the renal parenchyma. Biopsies, however, cannot be performed routinely in AKI because of their inappropriate risk-benefit ratio. Only recently, some attention has been placed on signs of structural damage to the nephrons, summarised in a meta-analysis of a novel renal marker, neutrophil gelatinase-associated lipocalin (NGAL) [8]. These concepts suggest that kidney damage or injury may occur, even in the absence of a parallel or consequent alteration of kidney function, and these changes are associated with the future development of clinical outcomes including prolonged length of stay, need for haemodialysis and mortality.

Human kidneys have a significant functional reserve and only when a significant number of nephrons are damaged is a meaningful reduction in GFR observed. A rise in SCr may in fact become evident only when $50 \%$ of the renal mass or more is compromised. In these circumstances, an insult that affects less than $50 \%$ of the nephrons may result in no change in $\mathrm{SCr}$ or only marginal changes in the baseline glomerular filtration rate. Thus, considerable proportions of patients are believed to incur subclinical AKI. The European Society of Urogenital Radiology (ESUR) Contrast Media Safety Committee recently updated CI-AKI guidelines with a retained definition: "CIN is a condition in which a decrease in renal function occurs within 3 days of the intravascular administration of a CM in the absence of an alternative aetiology. An increase in SCr by more than $25 \%$ or $44 \mu \mathrm{mol} / 1(0.5 \mathrm{mg} / \mathrm{dL})$ indicates CIN" [9]. Additionally, new AKI classifications such as the RIFLE [10] or AKIN [11] include, as minimal diagnostic criteria, changes in $\mathrm{SCr}$ as low as $0.3 \mathrm{mg} / \mathrm{dl}$, and validation studies demonstrated that such marginal conditions are associated with worse outcomes [12]. Although this is a refined approach, reinforced by the most recent Kidney Disease Improving Global Outcomes (KDIGO) recommendations, it still represents a functional criterion for AKI suggesting a sufficiently detectable decline in GFR, which may occur long after the injury has taken place [13]. The new KDIGO definition of AKI applies to CI-AKI and helps unify the clinical and research language around this common problem. This definition states AKI is defined as any of the following (not graded): an increase in $\mathrm{SCr}$ of $\geq 0.3 \mathrm{mg} / \mathrm{dl}(\geq 26.5 \mu \mathrm{mol} / \mathrm{l})$ within $48 \mathrm{~h}$, an increase in $\mathrm{SCr}$ to $\geq 1.5$ times the baseline that is known or presumed to have occurred within the prior 7 days, or urine volume $<0.5 \mathrm{ml} / \mathrm{kg} / \mathrm{h}$ for $6 \mathrm{~h}$. In addition, a severity of AKI stratified by three stages is shown in Table 1 .

Very recently, significant contributions to the literature have shown that we might be able to make an early diagnosis of AKI using a single structural or functional biomarker or a combination thereof capable of detecting kidney injury almost in real time [14]. Clinical validation studies have demonstrated the additional value of new biomarkers over standardised creatinine measurement not only for the possibility of an early diagnosis of AKI, but also for the detection 
Table 1 Kidney Disease International Global Outcomes (KDIGO) criteria for AKI

\begin{tabular}{lll}
\hline Stage & Serum creatinine & Urine output \\
\hline 1 & $1.5-1.9$ times baseline & $<0.5 \mathrm{ml} / \mathrm{kg} / \mathrm{h}$ for $6-12 \mathrm{~h}$ \\
& OR & \\
& $\geq 0.3 \mathrm{mg} / \mathrm{dl}(\geq 26.5 \mu \mathrm{mol} / \mathrm{l})$ & \\
increase & $<0.5 \mathrm{ml} / \mathrm{kg} / \mathrm{h}$ for $\geq 12 \mathrm{~h}$ \\
2 & $2.0-2.9$ times baseline & $<0.3 \mathrm{ml} / \mathrm{kg} / \mathrm{h}$ for $\geq 24 \mathrm{~h}$ \\
3 & 3.0 times baseline & OR \\
& OR & Anuria for $\geq 12 \mathrm{~h}$ \\
& Increase in serum creatinine & \\
& to $\geq 4.0 \mathrm{mg} / \mathrm{dl}(353.6 \mu \mathrm{mol} / \mathrm{l})$ & \\
& OR & \\
& Initiation of renal replacement & \\
therapy & \\
& OR, in patients $<18$ years, decrease & \\
in eGFR to $<35 \mathrm{ml} / \mathrm{min}$ per $1.73 \mathrm{~m}^{2}$ & \\
\hline
\end{tabular}

of a structural injury even if a kidney dysfunction or a rise in $\mathrm{SCr}$ will never become manifest $[15,16]$.

According to this modern approach, we might speculate that AKI could be diagnosed even in the absence of the classical signs that have characterised the syndrome in the past. Thus, a new category of AKI should be identified for those patients who display positivity of structural biomarkers even in the absence of creatinine rise as depicted in Table 2. According to this concept, patients undergoing imaging procedures requiring injection of contrast agents should be screened for both kidney damage and dysfunction in the period subsequent to the radiological investigation to detect structural and/or functional criteria for AKI. The fact that AKI is not clinically manifest according to classic criteria does not necessarily mean that the kidney is unharmed. A subclinical derangement may be unveiled by the new biomarkers and, although not responding to the classic criteria, it should be still defined as CI-AKI. To make a meaningful comparison, we may use the case of acute myocardial infarction where an increase in troponin, with at

Table 2 Conceptual framework for subclinical and clinically recognised CI-AKI

\begin{tabular}{lll}
\hline & $\begin{array}{l}\text { Functional criteria } \\
\text { (ESUR/RIFLE/AKIN/ } \\
\text { KDIGO) }\end{array}$ & $\begin{array}{l}\text { Structural criteria } \\
\text { (biomarkers) }\end{array}$ \\
\hline $\begin{array}{l}\text { No CI-AKI } \\
\begin{array}{l}\text { CI-AKI with structural } \\
\text { damage }\end{array}\end{array}$ & Negative & Negative \\
$\begin{array}{c}\text { CI-AKI with kidney } \\
\text { dysfunction }\end{array}$ & Positive & Negative \\
$\begin{array}{c}\text { CI-AKI with structural } \\
\text { damage and kidney } \\
\text { dysfunction }\end{array}$ & Positive & Positive \\
\hline
\end{tabular}

${ }^{a}$ These are all CI-AKIs for epidemiological and research purposes least one piece of supportive information, may be a sufficient trigger for a diagnosis and a therapeutic intervention even in the absence of classical signs and symptoms. In the field of radiology and especially of CI-AKI, a paradigm shift is likely to occur based on the abovementioned concepts. Future criteria may well integrate one or more injury markers with creatinine, urine output and/or other functional markers [17]. Today we do not have sufficient data to characterise severity based on structural biomarker levels and therefore the grading of the syndrome still relies on creatinine once dysfunction becomes manifest. However, we should consider the presence of a new class of AKI diagnosed by commercially available tubular damage biomarkers such as NGAL (blood and urine, Europe) and L-type fatty acidbinding protein (urine L-FABP, Japan) that may or may not evolve into a clinically manifest syndrome characterised by $\mathrm{SCr}$ rise and decreased GFR. Point-of-care testing both serum and urine before and after the imaging procedure could become a reality, allowing rapid and timely screening for CI-AKI. However, the scalability of this approach will be challenged in high-volume centres. On the horizon are additional markers including kidney injury molecule-1, a renal regenerative protein, and more pure markers of cellular damage such as alpha glutathione S-transferase (GST) and Pi GST. Thus, in the next few years we should be able to organise markers into a panel to provide inferences about reductions in renal filtration ( $\mathrm{SCr}$, cystatin-C), direct cellular damage (alpha and Pi GST), and cellular response to injury (NGAL to limit oxidative stress), including KIM-1 (to direct regeneration of tubules), hepatocyte growth factor, and other modulators of cell cycle proliferation and tissue recovery. As a general guide, all of these markers elevate chronically with CKD, and a doubling from baseline likely represents a significant change. Exact thresholds and detection limits have yet to be established in populations at risk with these markers. The field is likely to further develop and establish patterns of cell signalling (interleukin-18) and urinary losses of proteins by renal tubular cells that are associated with CI-AKI (N-acetylbeta-D-glucosaminidase, cystatin-C, albumin). However, these are less specific and unlikely to add more to the three major conceptual domains of filtration, damage and response to injury.

This new approach has several implications: (1) the diagnosis of AKI may include a larger spectrum of conditions and the epidemiology of the syndrome may significantly change with a greater incidence being recognised; (2) prevention and/or early organ protection strategies should be evaluated with newer diagnostic criteria for CI-AKI as an endpoint; (3) we should discriminate between acute kidney injury and acute renal dysfunction using the new staging 
classification (subclinical AKI for patients who are biomarker positive and creatinine negative). The diagnosis of subclinical AKI can be easily suspected today using available injury biomarkers. These tests are quite reliable with high sensitivity and specificity [18]. Sequential measurements and biomarkers curves may further help to identify trends characteristic of an isolated or an ongoing renal insult. This may open a new frontier in the diagnosis of $\mathrm{AKI}$ and its consequences in terms of prevention and therapeutic strategies. Data relevant to prevention and protection measures should be carefully evaluated in light of these new criteria. For example, volume expansion, iso-osmolar contrast [19], N-acetylcysteine and/ or bicarbonate infusion [20], and pre-emptive extracorporeal therapies [21] all have provided suggestive data in some studies for renal protection; however, the biochemical outcome, $\mathrm{SCr}$, is simply not sufficient. We strongly suggest that the definition of CI-AKI should be amended, including subclinical AKI as a true component of the contrast-induced kidney damage. New biomarkers should be incorporated in the criteria for diagnosis, and an integrated RIFLE or KDIGO (Table 2) should be utilised in future research.

In conclusion, we should not abandon creatinine or cystatin-C as biochemical reflections of renal filtration; however, we should combine them with markers of renal tubular injury. When subclinical AKI (elevation in a marker of injury but no change in $\mathrm{SCr}$ ) occurs after contrast exposure, it should be considered as CI-AKI. Although risk stratification, prevention measures and careful assessment of outcomes have globally reduced the rates of CI-AKI and improved patient comfort; particularly in the setting of acute myocardial infarction managed with percutaneous coronary intervention using intra-arterial contrast agents, there is a clear need for upgraded criteria to define AKI [19, 22, 23]. Current research looks forward to new diagnostic and therapeutic targets in this clinical domain, which will undoubtedly advance care in the specialties of radiology, cardiology, nephrology and other disciplines where intravascular iodine-based contrast agents are used.

Acknowledgements C. Ronco received speaker honoraria from Gambro, Merck, Astute, Alere Abbot and GE Healthcare. He is consulting for Asahi.

F. Stacul received speaker honoraria from Bracco and GE Healthcare

P.A. McCullough has no actual or potential conflict of interest.

\section{References}

1. McCullough PA, Sandberg KR (2003) Epidemiology of contrastinduced nephropathy. Rev Cardiovasc Med 4:S3-S9

2. Goldfarb S, McCullough PA, McDermott J, Gay SB (2009) Contrast-induced acute kidney injury: specialty-specific protocols for interventional radiology, diagnostic computed tomography radiology, and interventional cardiology. Mayo Clin Proc 84:170-179

3. McCullough PA, Adam A, Becker CR et al (2006) CIN Consensus Working Panel. Epidemiology and Prognostic Implications of Contrast-Induced Nephropathy. Am J Cardiol 98:5K-13K

4. Stolker JM, McCullough PA, Rao S et al (2010) Pre-procedural glucose levels and the risk for contrast-induced acute kidney injury in patients undergoing coronary angiography. J Am Coll Cardiol 55:1433-1440

5. Nyman U, Almén T, Jacobsson B, Aspelin P (2012) Are intravenous injections of contrast media really less nephrotoxic than intraarterial injections? Eur Radiol 22:1366-1371

6. Tumlin J, Stacul F, Adam A et al (2006) CIN Consensus Working Panel. Pathophysiology of Contrast-Induced Nephropathy. Am J Cardiol 98:14K-20K

7. Chvojka J, Sykora R, Krouzecky A et al (2008) Renal haemodynamic, microcirculatory, metabolic and histopathological responses to peritonitis-induced septic shock in pigs. Crit Care 12:R164

8. Haase M, Bellomo R, Devarajan P, Schlattmann P, Haase-Fielitz A (2009) NGAL Meta-analysis Investigator Group. Accuracy of neutrophil gelatinase-associated lipocalin (NGAL) in diagnosis and prognosis in acute kidney injury: A systematic review and meta-analysis. Am J Kidney Dis 54:1012-1024

9. Stacul F, van der Molen AJ, Reimer P et al (2011) Contrast induced nephropathy: updated ESUR Contrast Media Safety Committee guidelines. Eur Radiol 21:2527-2541

10. Bellomo R, Ronco C, Kellum JA, Mehta RL, Palevsky P (2004) Acute Dialysis Quality Initiative workgroup: Acute renal failure: definition, outcome measures, animal models, fluid therapy and information technology needs. The Second International Consensus Conference of the Acute Dialysis Quality Initiative (ADQI) Group. Crit Care 8:R204-R212

11. Mehta RL, Kellum JA, Shah SV et al (2007) Acute Kidney Injury Network. Acute Kidney Injury Network: report of an initiative to improve outcomes in acute kidney injury. Crit Care 11:R31

12. Chertow GM, Burdick E, Honour M, Bonventre JV, Bates DW (2005) Acute kidney injury, mortality, length of stay, and costs in hospitalized patients. J Am Soc Nephrol 16:3365-3370

13. Kidney disease: improving Global Outcomes Clinical Practice Guidelines on Acute Kidney Injury, http://www.kdigo.org/clinical_ practice_guidelines_3.php

14. Paragas N, Qiu A, Žhang Q et al (2011) The NGAL reporter mouse detects the response of the kidney to injury in real time. Nat Med 17:216-222

15. Haase M, Devarajan P, Haase-Fielitz A et al (2011) The outcome of neutrophil gelatinase-associated lipocalin-positive subclinical acute kidney injury: a multicenter pooled analysis of prospective studies. J Am Coll Cardiol 57:1752-1761

16. Nickolas TL, Schmidt-Ott KM, Canetta P et al (2012) Diagnostic and Prognostic Stratification in the Emergency Department Using Urinary Biomarkers of Nephron Damage. A Multicenter Prospective Cohort Study. J Am Coll Cardiol 59:246-255

17. Murugan R, Kellum JA (2011) Acute kidney injury: what's the prognosis? Nat Rev Nephrol 7:209-217

18. Ronco C, Cruz D, Noland BW (2012) Neutrophil GelatinaseAssociated Lipocalin Curve and Neutrophil GelatinaseAssociated Lipocalin Extended-Range Assay: a new biomarker approach in the early diagnosis of acute kidney injury and cardio-renal syndrome seminars in nephrology. Vol 32:pp $121-128$

19. McCullough PA, Brown JR (2011) Effects of Intra-Arterial and Intravenous Iso-Osmolar Contrast Medium (Iodixanol) on the Risk of Contrast-Induced Acute Kidney Injury: A Meta-Analysis. Cardiorenal Med 1:220-234 
20. Brown JR, Block CA, Malenka DJ, O'Connor GT, Schoolwerth AC, Thompson CA (2009) Sodium bicarbonate plus Nacetylcysteine prophylaxis: a meta-analysis. JACC Cardiovasc Interv 2:1116-1124

21. Cruz DN, Goh CY, Marenzi G, Corradi V, Ronco C, Perazella MA (2012) Renal replacement therapies for prevention of radiocontrast-induced nephropathy: a systematic review. Am J Med 125:66-78.e3
22. McCullough PA, Capasso P (2011) Patient discomfort associated with the use of intra-arterial iodinated contrast media: a metaanalysis of comparative randomized controlled trials. BMC Med Imag 24:11-12

23. Brown JR, McCullough PA, Splaine ME et al (2012) Northern New England Cardiovascular Disease Study Group. How do centres begin the process to prevent contrast-induced acute kidney injury: a report from a new regional collaborative. BMJ Qual Saf 21:54-62 